\title{
Improvement of Slippage and Wrinkling of Transporting Webs Using Micro-Grooved Rollers*
}

\author{
Shinji HIKITA $^{* *}$ and Hiromu HASHIMOTO ${ }^{* * *}$ \\ ${ }^{* *}$ FUJIFILM Corporation, \\ 210, Nakanuma, Minami-ashigara-shi, Kanagawa, 250-0193, Japan \\ E-mail: shinji_hikita@fujifilm.co.jp \\ *** Department of Mechanical Engineering, Tokai University, \\ 1117,Kitakaname, Hiratsuka-shi, Kanagawa,259-1292, Japan
}

\begin{abstract}
We describe an entirely new method of improving the slippage between web and roller. First, the concept of a micro-grooved roller is introduced, and then a theoretical model for estimating the slip onset velocity under the transport of web by the micro-grooved roller is formulated. The predicted results are compared with the experimental data to verify the applicability of the prediction model of slippage. Moreover, the web-wrinkling condition, which is in a trade-off relationship with the slippage condition, is also considered in the model. From the theoretical and experimental results, it is confirmed that the optimized micro-grooved roller is very effective in improving the slippage and wrinkling of thin web under the high-speed transport with low tension at the actual production line.
\end{abstract}

Key words: Web Handling, Slippage, Wrinkling, Micro-Grooved Roller, Optimum Design, Industrial Application

\section{Introduction}

The demand for liquid crystal displays (LCDs) in recent years is markedly increasing, whereas the price of LCD panels decreases. Therefore, a high productivity, more specifically a manufacturing technology that enables mass production at a low cost but a high quality, is required to produce high-functional webs, such as optical films used in LCDs. As concrete measures to increase the productivity, for example, the manufacturing speed of the conventional production lines should be increased, and thinner webs should be manufactured to reduce the cost of raw materials without losing the established high quality functions.

In the manufacturing process, webs are transferred by the traction between the web and roller. As the production speed increases, the amount of entrained air into the gap between the web and roller also increases, which is causing the traction loss and slippage. Slippage sometimes causes surface scratches and meandering of webs. Therefore, the manufactures of webs should avoid such web defects to keep the quality in the production lines. In particular, even a tiny scratch of micron scale is not permitted on an optical film for LCDs. To protect the traction loss due to air entrainment, it has been experimentally used to increase the web tension. However, when we want to avoid the slippage by this way, web wrinkling easily generate in the process, so it is required to discover the novel method for preventing both slippage and wrinkling simultaneously, which is typical trade-off problem in the web handling industries.

Until now, very few papers, treating such problem from the academic side, are available

*Received 29 July, 2009 (№. 09-0385) [DOI: 10.1299/jamdsm.4.226]

Copyright () 2010 by JSME 
in the literatures. ${ }^{(1)-(3)}$ Among them, Hashimoto ${ }^{(1)}$ originally demonstrated the idea of using the micro-grooved roller to improve the traction loss due to air entrainment and showed the effectiveness of the roller by the parametric study. However, the applicability of the micro-grooved roller to the actual web handling system was not verified experimentally. Regarding to the web wrinkling problems, Good et al. ${ }^{(2)}$ formulated the prediction model for generating the wrinkling of isotropic web. Hashimoto ${ }^{(3)}$ extended this model to the anisotropic webs and compared the predicted results with the measured data to verify the applicability of the model. But the trade-off relation between the slippage and wrinkling are not sufficiently clarified yet.

In this paper, the theoretical modeling for predicting the slippage and wrinkling is described for micro-grooved rollers and the optimization method for designing the effective micro-grooved rollers to prevent these web defects is formulated with experimental verifications. Moreover, an example of an application of this model to the actual web production line is introduced.

\section{Nomenclature}

\begin{tabular}{|c|c|}
\hline$a$ & inter-axial distance of the roller $(\mathrm{m})$ \\
\hline$b_{g}$ & groove width (m) \\
\hline$E_{x}$ & Young's modulus in the machine direction (GPa) \\
\hline$E_{z}$ & Young's modulus in the cross machine direction (GPa) \\
\hline$F$ & traction between the web and roller $(\mathrm{N})$ \\
\hline$f$ & bearing resistance of rotation $(\mathrm{N})$ \\
\hline$f(X)$ & objective function \\
\hline$g_{i}(\boldsymbol{X})$ & constraint functions $\quad(i=1 \sim 7)$ \\
\hline$h$ & air film thickness $(\mathrm{m})$ \\
\hline$h_{e q}$ & equivalent air film thickness (m) \\
\hline$h_{g}$ & groove depth $(\mathrm{m})$ \\
\hline$I^{\circ}$ & Inertia moment of the roller $\left(\mathrm{kg} \mathrm{m}^{2}\right)$ \\
\hline$n_{g}$ & number of micro-grooves \\
\hline$q_{x}$ & amount of air film flow $\left(\mathrm{m}^{3} / \mathrm{s}\right)$ \\
\hline$p_{c r}$ & critical pressure to prevent from scratch $(\mathrm{Pa})$ \\
\hline$p$ & pressure between the web and roller $(\mathrm{Pa})$ \\
\hline$R$ & roller radius $(\mathrm{m})$ \\
\hline$r$ & bearing radius $(\mathrm{m})$ \\
\hline$s$ & slip ratio \\
\hline$s_{g}$ & cross-sectional area of micro-groove $\left(\mathrm{m}^{2}\right)$ \\
\hline$T$ & inlet web tension $(\mathrm{N} / \mathrm{m})$ \\
\hline$T^{\prime}$ & outlet web tension $(\mathrm{N} / \mathrm{m})$ \\
\hline$T_{c r}$ & critical web tension to generate wrinkling $(\mathrm{N} / \mathrm{m})$ \\
\hline$T_{\text {slip }}$ & slip onset tension $(\mathrm{N} / \mathrm{m})$ \\
\hline$t_{w}$ & web thickness (m) \\
\hline$U$ & $\begin{array}{l}\text { combined velocity between the web and roller velocities }(\mathrm{m} / \mathrm{s}) \\
\left(=U_{w}+U_{r}\right)\end{array}$ \\
\hline$U_{w}$ & web velocity $(\mathrm{m} / \mathrm{s})$ \\
\hline$U_{r}$ & roller surface velocity $(\mathrm{m} / \mathrm{s})$ \\
\hline$U_{\text {slip }}$ & slip onset velocity $(\mathrm{m} / \mathrm{s})$ \\
\hline$W$ & web width (m) \\
\hline $\boldsymbol{X}$ & design variable vector \\
\hline$\eta$ & air viscosity (Pa s) \\
\hline$\Theta$ & wrap angle (rad) \\
\hline
\end{tabular}




\begin{tabular}{|c|c|}
\hline$\theta$ & misalignment angle of the roller (rad) \\
\hline$\theta_{c r}$ & critical misalignment angle of the roller (rad) \\
\hline$\mu_{\text {eff }}$ & effective friction coefficient including air film effect \\
\hline$\mu_{\text {slip }}$ & friction coefficient at the onset of slippage \\
\hline$\mu_{s}$ & static friction coefficient between the web and roller \\
\hline$v_{x}, v_{z}$ & Poisson's ratio of the web in the machine and cross machine directions \\
\hline$\rho$ & density of air $\left(\mathrm{kg} / \mathrm{m}^{3}\right)$ \\
\hline$\sigma$ & combined $r m s$ roughness between the web and roller $(\mathrm{m})$ \\
\hline$\sigma_{z c r}$ & critical buckling stress in the cross machine direction $(\mathrm{Pa})$ \\
\hline$\sigma_{x}$ & normal stress in the machine direction $\left(=T / t_{w}\right)(\mathrm{Pa})$ \\
\hline$\dot{\omega}$ & angular acceleration of the roller $\left(\mathrm{rad} / \mathrm{s}^{2}\right)$ \\
\hline
\end{tabular}

\section{Air Film Thickness Estimation for Micro-Grooved Roller}

\subsection{Air film thickness estimation for non-grooved roller}

For the purpose of improving the traction characteristics between the web and roller, it is needed first to predict the entrained air film thickness. Before formulating the micro-grooved roller theory to predict the traction between the web and roller, the traditional foil bearing model, that gives the simplified formula for evaluating the entrained air film thickness between the web and non-grooved roller, is described briefly in the following.

In the foil bearing model as shown in Fig. 1, the width of web $W$ is generally larger than the roller diameter $2 R$, and then the air film flow can be treated as one-dimensional in the $x$-direction. Under such situation, the nonlinear Reynolds equation and the web elastic equation are given, respectively as follows.

$$
\begin{gathered}
\frac{d}{d x}\left(h^{3} p \frac{d p}{d x}\right)=6 \eta U \frac{d(p h)}{d x} ; U=U_{r}+U_{w} \\
\frac{E t_{w}{ }^{3}}{12\left(1-v_{x}{ }^{2}\right)} \frac{d^{4} h}{d x^{4}}+\frac{T}{R}\left(1-R \frac{d^{2} h}{d x^{2}}\right)=p-p_{a}
\end{gathered}
$$

In the case of very thin webs treated in this paper, the first term of the left hand side in Eq. (2) can be neglected, and Eq. (2) is reduced to the following equation.

$$
\frac{T}{R}\left(1-R \frac{d^{2} h}{d x^{2}}\right)=p-p_{a}
$$

Solving Eqs. (1) and (3) simultaneously with the proper boundary conditions on $p$ and $h$, the pressure and air film thickness distributions are obtained as shown in Fig. 2, in which the non-dimensional quantities are introduced as follows.

$$
p^{*}=\frac{p}{T / R}, \quad h^{*}=\frac{h}{R \varepsilon^{2 / 3}}, \quad \varepsilon=\frac{6 \eta U}{T}, \quad \beta=\frac{\Theta}{\varepsilon^{1 / 3}}
$$

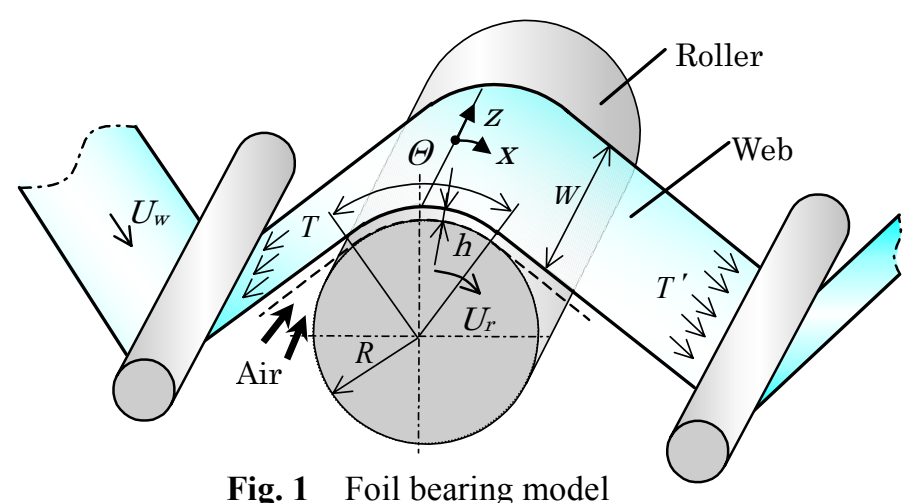

Fig. 1 Foil bearing model 


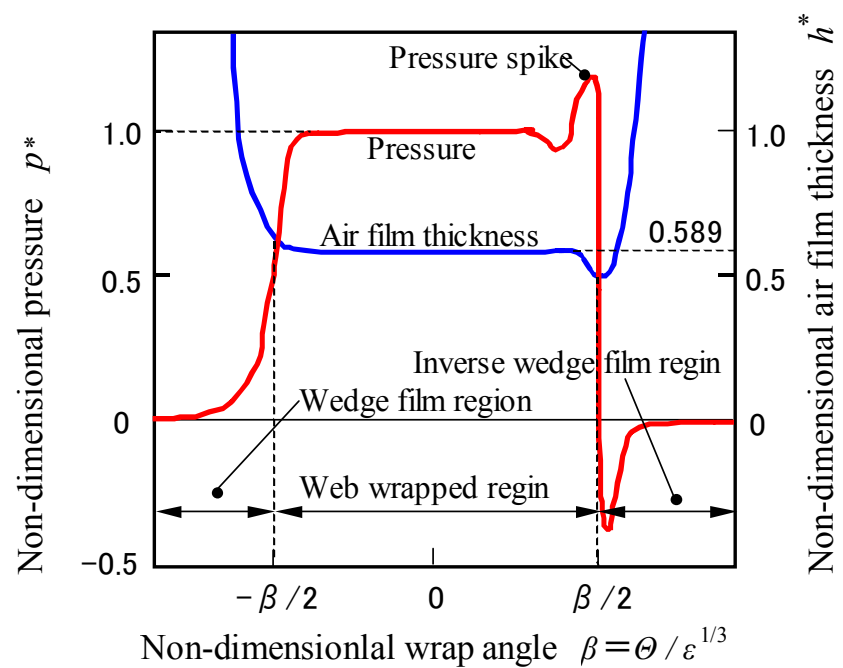

Fig. 2 Air film thickness and pressure distributions for non-grooved roller

As can be seen in the figure, in the web wrapped region the non-dimensional pressure $p^{*}$ becomes the constant value of $p^{*} \cong 1$ and the non-dimensional air film thickness $h^{*}$ also becomes the constant value of $h^{*} \cong 0.589$, respectively, which are independent of the non-dimensional wrap angle $\beta$, and it was confirmed that such a trend does not change for a wide range of web wrap angle $\Theta$ larger than $0 \mathrm{deg}$. From this fact, the dimensional pressure $p$ and air film thickness $h$ between the web and non-grooved roller are approximately expressed as follows.

$$
\begin{gathered}
p=\frac{T}{R} \\
h=0.589 R\left(\frac{6 \eta U}{T}\right)^{\frac{2}{3}}
\end{gathered}
$$

Moreover, neglecting the pressure gradient flow in the web wrapped region, the air flow rate per unit width between the web and non-grooved roller is given as follows.

$$
q_{x}=\frac{\rho h U}{2}
$$

\subsection{Air film thickness estimation for micro-grooved roller}

The concept of micro-grooved roller was introduced originally by Hashimoto ${ }^{(1)}$. The micro-grooved roller presented in this paper is manufactured a number of micro-size grooves in the circumferential direction of roller surface as shown in Fig. 3. In the micro-size grooves, it is considered that the air flow is dominated by the viscous flow and the pressure gradient flow in the web wrapped region can be neglected as in the case of non-grooved roller. Based on such considerations, the air flow rate per unit width between the web and micro-grooved roller is expressed as follows.

$$
q_{x}=\frac{\rho U}{2}\left(h+\frac{n_{g} s_{g}}{W}\right)
$$

Introducing the equivalent air film thickness $h_{e q}$ as shown Fig. 3, the air flow rate is expressed directly with Eq. (7) as follows.

$$
q_{x}=\frac{\rho U}{2} h_{e q}=0.589 \frac{\rho R U}{2}\left(\frac{6 \eta U}{T}\right)^{\frac{2}{3}}
$$




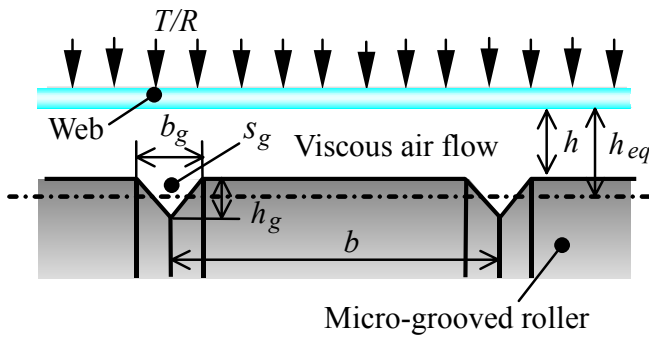

Fig. 3 Concept of micro-grooved roller

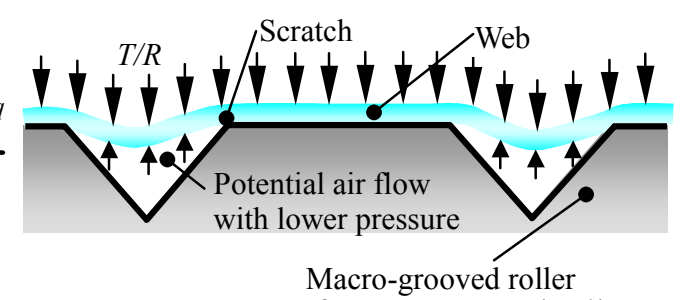

Fig. 4 Concept of macro-grooved roller

Equating Eqs. (8) and (9), the air film thickness, $h$, between the web and the top surface of micro-grooved roller, shown in Fig. 3, is obtained as follows.

$$
h=0.589 R\left(\frac{6 \eta U}{T}\right)^{\frac{2}{3}}-\frac{n_{g} s_{g}}{W}
$$

It follows from Eq. (10) that the air film thickness $h$ can be reduced effectively by suitable designing the groove size, $n_{g}$ and $s_{g}$. When the air film thickness $h$ becomes negative, it should be equated to zero.

The idea of grooved roller itself has been well known for the web handling engineers, but in that case, the groove size is millimeter scale, and therefore we call such type of groove as "macro-groove". As far as the authors know, however, the idea of "micro-groove" to improve the traction characteristics between the web and roller has not been known, except the report by Hashimoto et al. ${ }^{(1)}$.

Figure 4 shows the conceptual diagram of macro-grooved roller. As the size of macro-grooves is usually millimeter scale as mentioned above, the air flow inside the groove is dominated by the potential flow. In this case, the air flow through the macro-grooves is accelerated and the pressure inside the groove becomes lower than that outside. As a result, the web is easy to deform and sometimes generate scratches as shown in the figure. On the other hand, the pressure inside the micro-groove does not become lower, because the air flow inside the groove is dominated by the viscous flow and adhered to the groove wall, and therefore is not accelerated.

\section{Prediction of Slippage and Wrinkling}

Figure 5 shows the conceptual diagram of the interface between the web and roller. The effective friction coefficient $\mu_{e f f}$, which is including the effect of entrained air as mentioned in the former chapter, is approximately given as follows.

$$
\mu_{e f f}=\left\{\begin{array}{lc}
\mu_{s} & (h<\sigma) \\
\frac{\mu_{s}}{2}\left(3-\frac{h}{\sigma}\right) & (\sigma \leq h \leq 3 \sigma) \\
0 & (h>3 \sigma)
\end{array}\right.
$$

where $h$ is the entrained air film thickness given by Eq. (10), and $\sigma$ is the rms composite surface roughness between the web and roller, which is defined in following.

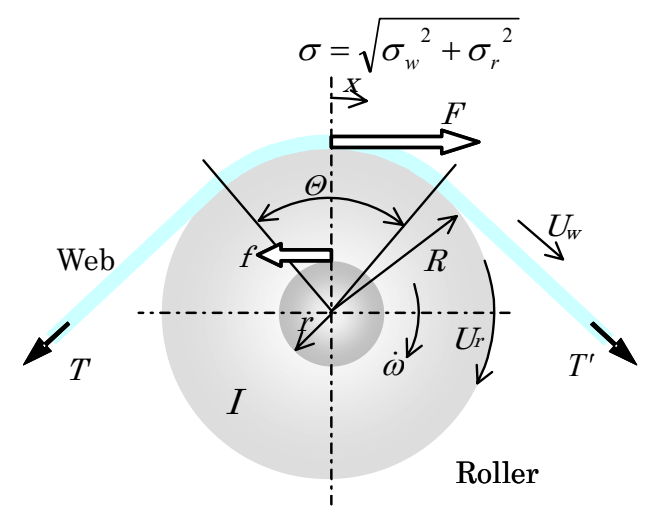

Fig. 5 Traction transmitted from roller to web or from web to roller 
From Fig. 5, the slip onset condition between the web and roller is expressed as follows;

$$
F R=I \dot{\omega}+f r
$$

where $I \dot{\omega}$ means the inertia torque that can be neglected under the steady state condition.

The traction, $F$, transmitted between the web and roller is given by the following relation.

$$
F=W\left(T^{\prime}-T\right)
$$

From the Euler's belt theory, the relation between $T$ and $T^{\prime}$ is given as follows.

$$
T^{\prime}=T \mathrm{e}^{\mu_{e f f} \Theta}
$$

From Eq. (13) through Eq. (15), the effective friction coefficient to start the slippage is given as follows.

$$
\mu_{\text {slip }}=\frac{1}{\Theta} \ln \left(1+\frac{I \dot{\omega}+f r}{W R T}\right)
$$

Generally, it is considered that the slippage will occur in the mixed lubrication region of $\sigma \leq h \leq 3 \sigma$, and then equating Eq. (16) to the middle expression in Eq. (11) after substituting $h$ from Eq. (10), the slip onset velocity of roller for the fixed value of tension is obtained by the following equation. At the same time, the slip onset tension for the fixed value of velocity is also obtained by solving the equation with respect to tension, $T$.

$$
U=\frac{1}{12}\left\{5.093-\frac{3.396}{\mu_{s} \Theta} \ln \left(1+\frac{I \dot{\omega}+f r}{W R T}\right)+1.698 \frac{n_{g} s_{g}}{\sigma W}\right\}^{\frac{3}{2}} \frac{T}{\eta}\left(\frac{\sigma}{R}\right)^{\frac{3}{2}}
$$

When the micro-grooved roller is used for the transport of web, the traction between the web and roller will be greatly improved even in the case of high speed transport with low tension. However, it has been demonstrated by $\operatorname{Good}^{(2)}$ and Hashimoto ${ }^{(3)}$ that the wrinkles as shown in Figs. 6(a) and 6(b) will be generated due to the misalignment of roller when the sufficient level of friction coefficient is maintained between the web and roller. Especially, in the case of very thin web, like the optical LCD film, the wrinkling is easy to generate and it should be prevented in the production lines.

According to Hashimoto ${ }^{(3)}$, in the mechanism of generating wrinkles, the trough is generated first in the transported web between roller and roller, and after that it leads to wrinkles on the surface of the down stream roller surface, as shown in Fig. 6(b). In this paper, the following prediction model ${ }^{(3)}$ developed for the non-grooved roller is also applied to predict the onset of wrinkling for the micro-grooved roller.

Based on the linearized buckling theory and the energy method, the critical buckling stress in the cross machine direction of the transported web between roller and roller as shown in Fig. 7(a) is given by the following equation.

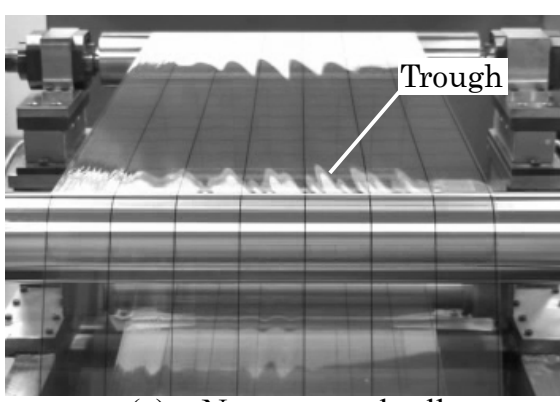

(a) Non-grooved roller

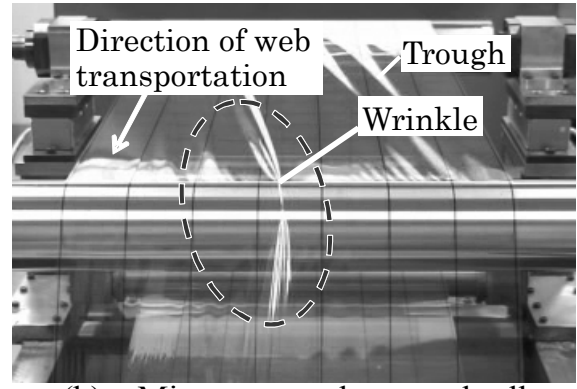

(b) Micro-grooved grooved roller

Fig. 6 Observation of wrinkle generation $\left(U_{w}=0.15 \mathrm{~m} / \mathrm{s}, W=0.3[\mathrm{~m}], \Theta=60[\mathrm{deg}], R=0.04[\mathrm{~m}], T=150[\mathrm{~N} / \mathrm{m}], \theta=0.2[\mathrm{deg}]\right)$ 


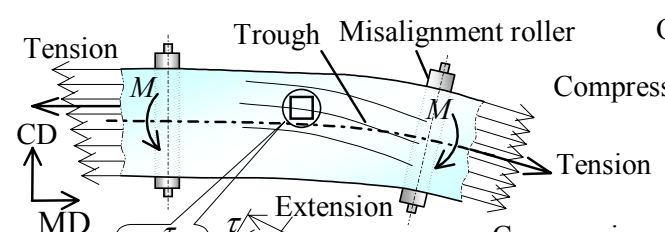

Onset of wrinkling Misalignment roller

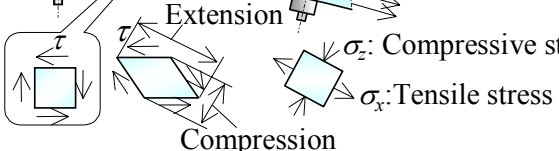

$\sum_{\sigma_{x}}^{\sigma_{z}: \text { Tensile stress }}$

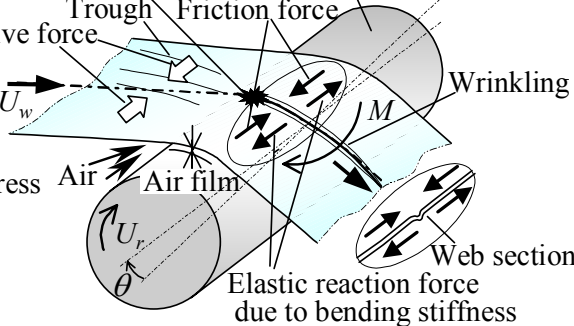

(a) Mechanism of trough generation between roller and roller due to roller misalignment (1st step)

(b) Mechanism of onset of wrinkling from trough on the roller surface (2nd step)

Fig. 7 Mechanism of wrinkling generation

$$
\sigma_{z c r}=\frac{W^{2}}{i^{2} a^{2}}\left\{\sigma_{e}\left(1+\zeta_{1} i^{4} \frac{a^{4}}{W^{4}}+\zeta_{2} i^{2} \frac{a^{2}}{W^{2}}\right)-\sigma_{x}\right\}
$$

where the coefficients $\zeta_{1}$ and $\zeta_{2}$ and the stresses $\sigma_{x}$ and $\sigma_{e}$ are defined, respectively, as follows;

$$
\zeta_{1}=\frac{E_{z}}{E_{x}}, \quad \zeta_{2}=\frac{4\left(1-v_{x} v_{z}\right)}{1+v_{x}+\left(1+v_{z}\right) / \zeta_{1}}+v_{z}+v_{x} \zeta_{1}, \quad \sigma_{x}=\frac{T}{t_{w}}, \quad \sigma_{e}=\frac{\pi^{2} E_{x} t_{w}{ }^{2}}{12 a^{2}\left(1-v_{x} v_{z}\right)}
$$

and $i$ means the corrugation number to be satisfied with the following inequality.

$$
\sigma_{e}\left\{1-i^{2}(i+1)^{2} \zeta_{1} \frac{a^{4}}{W^{4}}\right\}<\sigma_{x}<\sigma_{e}\left\{1-(i-1)^{2} i^{2} \zeta_{1} \frac{a^{4}}{W^{4}}\right\}
$$

When the misalignment angle of the roller, $\theta$, exceeds the critical angle, the trough as shown in Fig. 6 will be generated, and the wrinkling onset condition is expressed as follows.

$$
\theta \geq \theta_{c r}=\frac{6 a^{2}}{E_{x} W^{2}} \sqrt{\sigma_{z c r}^{2}-\sigma_{z c r} \sigma_{x}}
$$

Since the web wrapped over the roller takes the cylindrical form as shown in Fig. 7(b), the critical buckling stress on the roller is given as follows.

$$
\sigma_{z c r}=\frac{t_{w}}{R} \sqrt{\frac{E_{x} E_{z}}{3\left(1-v_{x} v_{z}\right)}}
$$

The compressive stress becomes zero at the edges of web width and increases towards the web center with a gradient determined by traction, and then the trough will grow up and leads to generate wrinkling on the center line of web wrapped over the roller, if the following condition is satisfied.

$$
\sigma_{z c r}=\mu_{e f f} \frac{T_{c r}}{R} \frac{W}{2 t_{w}}
$$

Substituting Eq. (22) into Eq. (23), the wrinkling onset condition with respect to web tension is obtained as follows.

$$
T \geq T_{c r}=\frac{2 t_{w}{ }^{2}}{\mu_{e f f} W} \sqrt{\frac{E_{x} E_{z}}{3\left(1-v_{x} v_{z}\right)}}
$$

When Eqs. (21) and (24) are satisfied simultaneously, the wrinkles will generate on the center line of web wrapped over the roller.

\section{Optimum Design of Micro-Groove Size}

Figure 8 shows one of examples of the predicted results of the slippage and wrinkling regions obtained by the above introduced theory for both cases of non-grooved and micro-grooved rollers. The critical web tension to start the slippage becomes much smaller 


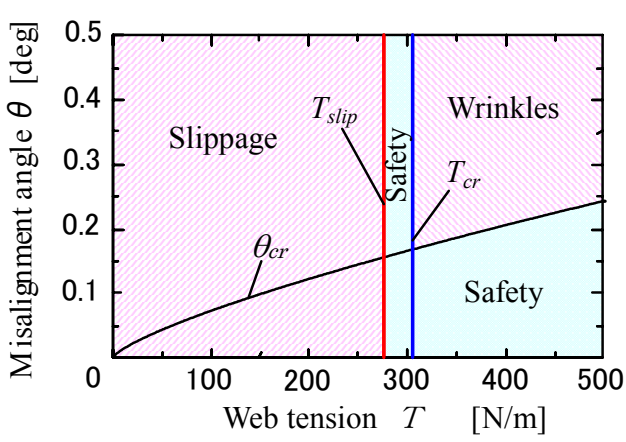

(a) Non-grooved roller

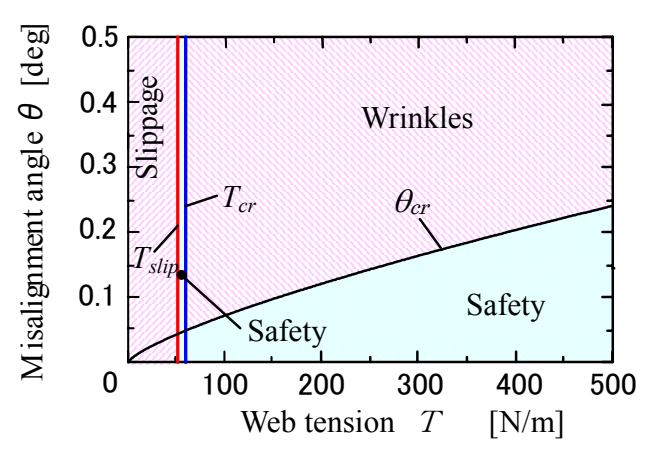

(b) Mircro-grooved Roller $1(\mathrm{~b}=6[\mathrm{~mm}])$

Fig. 8 Relation between slippage, wrinkling and safety regions $\left(U_{r}=0.3[\mathrm{~m} / \mathrm{s}], W=0.3[\mathrm{~m}], \Theta=60[\mathrm{deg}], R=0.04[\mathrm{~m}], a=0.8[\mathrm{~m}]\right)$

in the case of micro-grooved roller for the fixed value of the roller velocity, and it means that the web can be transferred successfully with low tension. However, the critical web tension to generate wrinkling also becomes smaller, and as a result, the safety operation region of micro-grooved roller becomes narrower than that of non-grooved roller. To solve such a serious trade-off problem, it is required to optimize the micro-groove sizes by the following way.

Find $\boldsymbol{X}$ to minimize $f(\boldsymbol{X})$ subject to $g_{i}(\boldsymbol{X}) \leq 0$

where the design vector $\boldsymbol{X}$, objective function $f(\boldsymbol{X})$ and the constraint functions $g_{\mathrm{i}}(\boldsymbol{X})$ are defined, respectively, as follows.

$$
\begin{gathered}
\boldsymbol{X}=\left(b_{g}, h_{g}, n_{g}\right) \\
f(X)=\frac{T_{\text {slip }}}{T_{c r}} \\
g_{1}(\boldsymbol{X})=b_{g}-b_{g \text { min }}, \quad g_{2}(\boldsymbol{X})=b_{g \text { max }}-b_{g} \\
g_{3}(\boldsymbol{X})=h_{g}-h_{g \text { min }}, \quad g_{4}(\boldsymbol{X})=h_{g \text { max }}-h_{g} \\
g_{5}(\boldsymbol{X})=n_{g}-n_{g \text { min }}, \quad g_{6}(\boldsymbol{X})=n_{g \max }-n_{g} \\
g_{7}(\boldsymbol{X})=\frac{T}{R\left(1-n_{g} b_{g} / W\right)}-p_{c r}
\end{gathered}
$$

In Eqs. (27) and (28), $T_{\text {slip }}$ means the slip onset tension obtained from Eq. (17) and the last constraint function $g_{7}(X) \leq 0$ is imposed to protect the scratches due to high pressure at the top surface of grooves and groove corners, in which $p_{c r}$ means the critical pressure to generate the scratches.

\section{Experiments}

To verify the applicability of prediction models proposed in this paper, the experiments on the generation of slippage and wrinkling were carried out for five types of rollers, one of which is the non-grooved roller, and the others are micro-grooved rollers. These micro-grooved rollers were designed and manufactured based on the micro-grooved roller theory developed in the former chapters including the optimum design. The upper and lower limits of $b_{g}, h_{g}, n_{g}$ and the critical value of $p_{c r}$ in Eq.(28) were predetermined as $b_{\text {gmin }}=100 \mu \mathrm{m}, b_{g \max }=500 \mu \mathrm{m}, h_{g \min }=50 \mu \mathrm{m}, h_{g \max }=300 \mu \mathrm{m}, n_{g \min }=30, n_{\text {gmax }}=400$ and $p_{c r}=1.5 \mathrm{kPa}$, respectively. The dimensions of designed micro-grooves are listed in Table 1, in which the cross section of groove was selected as triangle. 
Table 1 Dimension of test rollers

\begin{tabular}{llllccccc}
\hline \hline \multicolumn{2}{c|}{ Item } & & & Non-grooved & Groove1 & Groove 2 & Groove 3 & Optimized \\
\hline Groove pitch & $b$ &, & $\mathrm{~mm}$ & - & 6.0 & 3.0 & 2.1 & 1.0 \\
Groove width & $b_{g}$ &, & $\mu \mathrm{m}$ & - & 200 & 200 & 300 & 460 \\
Groove depth & $h_{g}$ &, & $\mu \mathrm{m}$ & - & 100 & 100 & 100 & 200 \\
Groove number & $n_{g}$ & & - & 51 & 101 & 143 & 301 \\
Roller roughness & $\sigma_{r}$, & $\mu \mathrm{m}$ & 0.3 & 0.3 & 0.3 & 0.3 & 0.3 \\
\hline \hline
\end{tabular}

In the experiment on slippage, the experimental apparatus as shown in Fig. 9 was used. One end of test web was glued to the other end over five rollers to form a loop that enables the endless transport of web. The web tension was set up step by step in the wide range of tension from $T=10 \mathrm{~N} / \mathrm{m}$ to $T=210 \mathrm{~N} / \mathrm{m}$. The roller surface velocity was continuously changed from $U_{r}=0$ to $U_{r}=5 \mathrm{~m} / \mathrm{s}$.

The web velocity and the roller surface velocity were, respectively, measured by the laser doppler velocimeter (LDV) at the same time, and after measuring these velocities the slip ratio defined by the following equation was calculated immediately.

$$
s=\frac{\left|U_{r}-U_{w}\right|}{U_{r}}
$$

If the obtained slip ratio was larger than 0.01 , we have judged the slippage was occurred between the web and roller, and the roller surface velocity at that time was defined and recorded as the slip onset velocity $U_{\text {slip }}$.

In the experiment on wrinkling, the same experimental apparatus as shown in Fig. 9 was used, but the test roller was misaligned continuously from $\theta=0$ to $\theta=1.5 \mathrm{deg}$ in the horizontal plane, using a micro-screw until the wrinkling was completely generated in the web. The wrinkles generated on the roller surface were permanent deformation and did not recover, but the trough generated between the roller and roller was elastic deformation due to buckling and it could be recovered. Therefore, it was not difficult to distinguish the trough and wrinkling during the experiments.

When the wrinkling was occurred, the critical misalignment angle of roller, $\theta_{c r}$, was immediately measured and recorded.

The physical properties of tested web are listed in Table 2 .

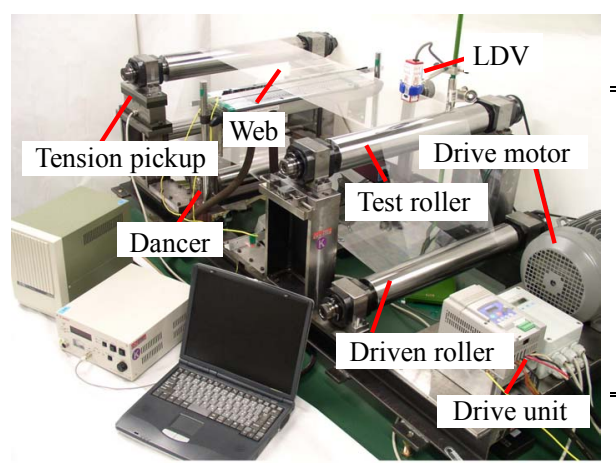

Table 2 Physical properties of tested web

\begin{tabular}{lll|c}
\hline \hline Young's modulus in MD & $E_{x}$, & $\mathrm{Gpa}$ & 4.37 \\
Young's modulus in CD & $E_{z}$, & $\mathrm{Gpa}$ & 4.29 \\
Poisson's ratio in MD & $v_{x}$ & & 0.3 \\
Poisson's ratio in CD & $v_{z}$ & & 0.3 \\
Static friction coefficient & $\mu_{s}$ & 0.3 \\
Web thickness & $t_{w}, \mu \mathrm{m}$ & 25 \\
Web $r m s$ roughness & $\sigma_{w}, \mu \mathrm{m}$ & 0.032 \\
\hline \hline
\end{tabular}

Fig. 9 Test apparatus for slippage and wrinkling

\section{Results and Discussion}

Figure 10 shows the relation between the slip onset velocity $U_{\text {slip }}$ and web tension $T$ for the fixed values of $W=0.3 \mathrm{~m}, \Theta=60 \mathrm{deg}, R=0.04 \mathrm{~m}, \mathrm{a}=0.8 \mathrm{~m}$ and $\theta=0$ (aligned roller) in which the plots show the measured data and the thick lines show the predicted results by Eq. (17), respectively. The upper region of each line is corresponding to the slippage region and the lower region is corresponding to the non-slippage region, respectively. As can be seen in the 


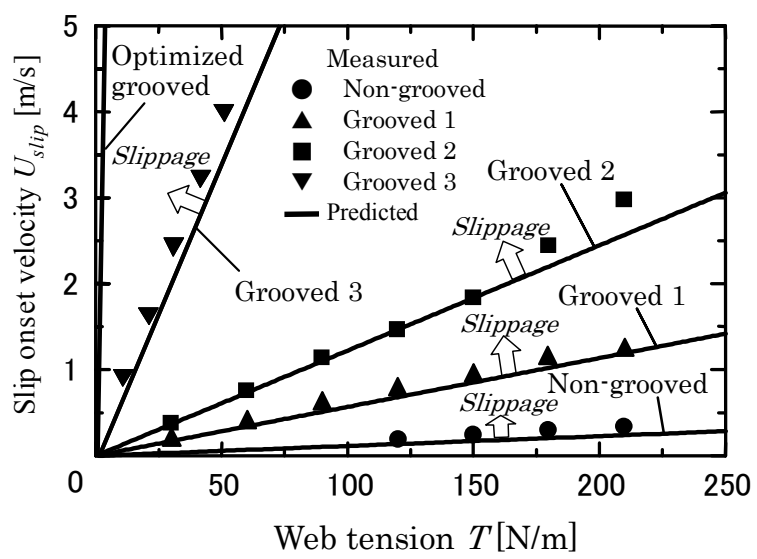

Fig. 10 Relation between slip onset velocity and web tension $(W=0.3[\mathrm{~m}], \Theta=60[\mathrm{deg}], R=0.04[\mathrm{~m}], a=0.8[\mathrm{~m}], \theta=0)$

figure, the slip onset velocity of micro-grooved roller with smaller groove pitch becomes higher than that of non-grooved roller. Especially, in the case of the optimized micro-grooved roller, no slippage and scratch could be seen during the experiments. The slip onset velocity increases drastically even in the case of very low tension. As a result, the micro-grooved roller, especially the optimized micro-grooved roller, is very much effective to prevent the slippage and scratches between the web and roller under the high transport speed with low web tension. In the figure, the predicted results agree well with the measured data, and then the applicability of the micro-grooved roller theory proposed in this paper is verified experimentally.

Figure 11 shows the theoretical prediction of the slippage region, wrinkling region and the safety operation region without both slippage and wrinkling accompanied with the measured data, in which the black plots mean the generation of wrinkling and the white plots mean the onset of slippage, respectively. It should be noticed that the horizontal axis (web tension) in Fig. 11(b) through 11(d) is half scale of that in Fig. 11(a), and the calculated value of the critical misalignment angle $\theta_{c r}$ is the same for all cases. As can be seen in the figure, the slip onset tension $T_{\text {slip }}$ of the micro-grooved roller decreases considerably and the slippage region becomes smaller with a decrease of groove pitch, especially the slip onset tension of the optimized micro-grooved roller approaches zero and there is no slippage region.

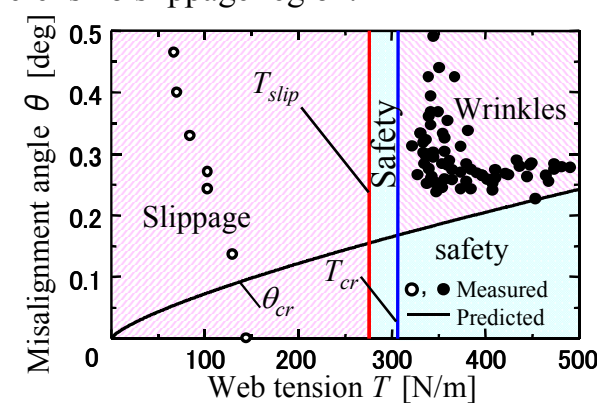

(a) Non-grooved roller

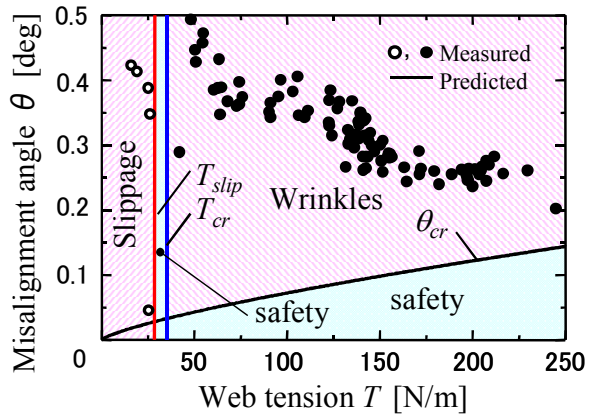

(c) Grooved roller 2 ( $b=3[\mathrm{~mm}])$

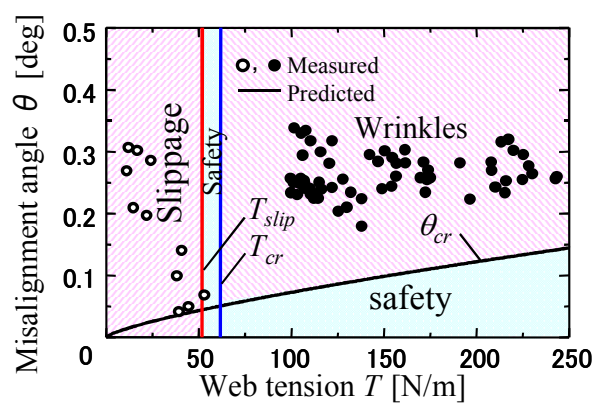

(b)Grooved roller 1 ( $b=6[\mathrm{~mm}])$

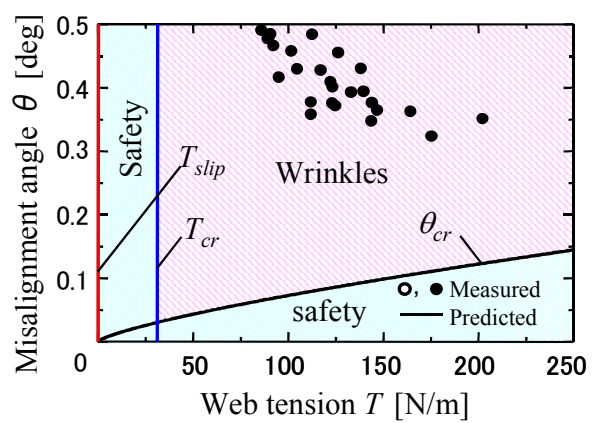

(d) Optimized grooved roller ( $b=1[\mathrm{~mm}]$ )

Fig. 11 Predicted and measured results of wrinkling and slippage $\left(U_{r}=0.3[\mathrm{~m} / \mathrm{s}], W=0.3[\mathrm{~m}], \Theta=60[\mathrm{deg}], R=0.04[\mathrm{~m}], a=0.8[\mathrm{~m}]\right)$ 
On the other hand, the critical tension to start the wrinkling $T_{c r}$ of the micro-grooved roller also decreases and wrinkling region becomes larger with a decrease of groove pitch. As a result, the safety operation region becomes smaller as shown in Figs 11(b) and 11(c). This fact shows the strong trade-off relation between the slippage and wrinkling, so it is required to optimize the micro-groove sizes to enlarge the safety operation region as much as possible. Figure. 11(d) shows the effectiveness of the optimized grooved roller that was designed by the optimization method proposed in this paper. In this case, the safety operation region becomes much larger than the other micro-grooved rollers shown in Figs.11 (b) and 11(c). Especially at the conditions of high speed and low tension, which is the present and future trend in the handling of very thin, high functional webs, the effectiveness of the optimized micro-grooved roller is remarkable compared to the other rollers including non-grooved roller. There are reasonable agreements between the predicted results and the measured data.

\section{Application to Production Line}

In the actual production lines of webs, many rollers are used for the transport of webs. Normally bearing torque is designed as much smaller than the traction torque, because the tension at the down stream of web line becomes much larger than that at the up stream, even if each bearing torque of rollers is less than $10^{-2} \mathrm{Nm}$. At this condition thin web will be expanded in the machine direction. In the present experiments carried out on the actual production lines, some break torque was added to the non-driven roller, and under the condition that the roller velocity was just $99.7 \%$ of the web velocity, namely slip ratio $s=$ 0.003 , break torque $f r$ was measured as slippage torque. From the measured value of $f r$, and predetermined values of web tension $T$, and wrap angle $\Theta$, the effective friction coefficient $\mu_{\text {eff }}$ was calculated by Eq. (16). Figure 12 shows a comparison between the friction coefficient for the optimized micro-grooved roller and those for the non-optimized micro-grooved and non-grooved rollers. The optimized micro-grooved roller in the production line has almost the same groove sizes of the optimized micro-groove as shown in Table 1, although the triangle groove shape and surface roughness are different. The optimized micro-grooved roller exhibits a high friction coefficient, and it causes no slippage even at an increased roller velocity with very low tension of $40 \mathrm{~N} / \mathrm{m}$. On the other hand, the friction coefficient for the non-optimized micro-grooved roller decreases as the web velocity increases. As can be seen in the figure, the optimized micro-grooved roller is very effective in the actual production line of webs.

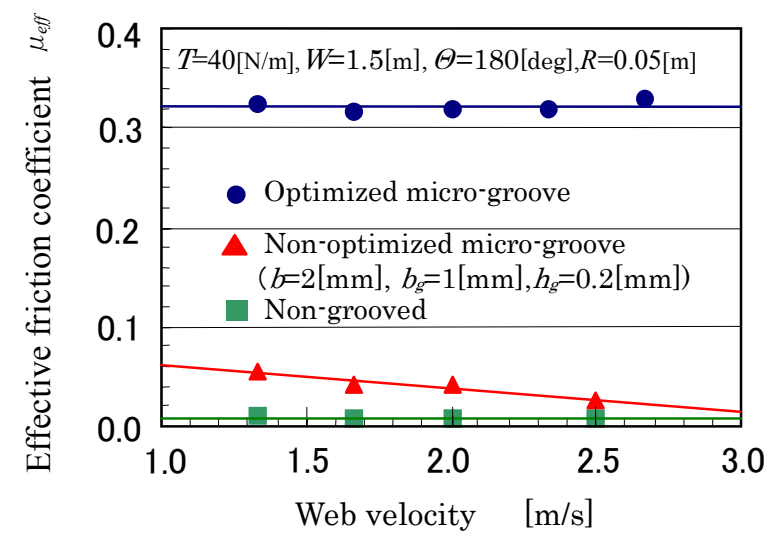

Fig. 12 Effective friction coefficients for three types of rollers in production line

On the other hand, in the actual production lines of webs, in order to increase the slip onset velocity, the non-driven rollers fabricated as light as possible are generally used to minimize resistance torque, which is equivalent to $I \dot{\omega}+f r$ as already introduced in Eq. 
(13). However, when the weight of roller is reduced, the roller rigidity decreases at the same time and the roller will be bended by the tension, which leads to the generation of wrinkles similar to the wrinkles due to the misalignment of roller as already shown in Fig. 6. To avoid such type of wrinkles, it has been well known experimentally to use the concave roller as shown in Fig. 13, because the concave roller has a function to expand the web in the axial direction of roller. Therefore, combining the idea of micro-groove and concave roller, which means the use of the concave roller with micro-grooves, we can expect to prevent both slippage and wrinkling simultaneously even in the case of very high speed and low tension transport of webs.

Figure 13 shows the evidence of the effectiveness of the concave roller with optimized micro-grooves in the actual production line of webs. As can be seen in the figure, the wrinkling onset tension for the concave roller with the optimized micro-grooves became double compared to that for the straight roller with the same size of grooves. At that time, no slippage could be seen between the web and roller. From this fact, it was confirmed that combining the concave roller and the optimized micro-groove is very effective to transport the web without slippage and wrinkling under high velocity and low tension.

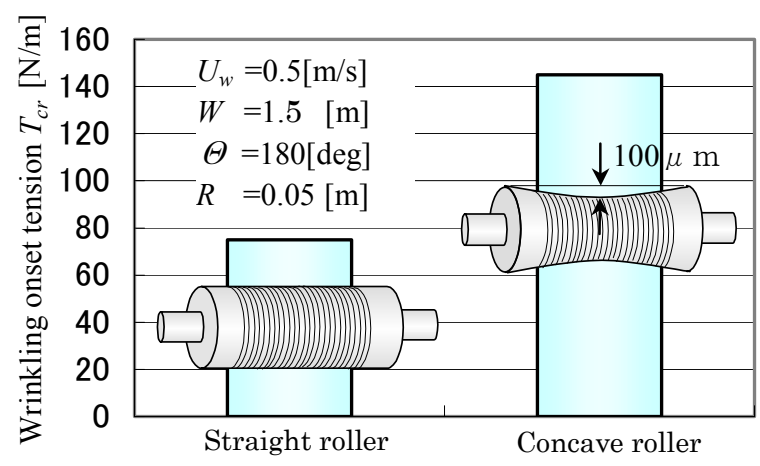

Fig. 13 Comparison of wrinkling onset tensions in production line

\section{Conclusion}

In this study, a theoretical model for predicting slippage and wrinkling between the web and micro-grooved roller was newly formulated, and the effectiveness of the model was verified experimentally. The main conclusions are summarized as follows.

(1) The results based on the theoretical model for predicting the onset of slippage between the web and micro-grooved roller agree well with the experimental results.

(2) The results based on the theoretical model for predicting the onset of wrinkling reasonably agree with the experimental results.

(3) It was verified theoretically and experimentally that the optimized micro-grooved roller enables to enlarge the safety transport region without slippage and wrinkling.

(4) Combining the concave roller and the optimized micro-groove, the safety transport region of webs is remarkably enlarged compared to the case of conclusion (3).

\section{References}

(1) Hashimoto, H., and Nakagawa, H., "Improvement of Web Spacing and Friction Characteristics by Two Types of Stationary Guides", Trans. ASME, Journal of Tribology, Vol. 123, 2001, pp.509-516.

(2) Good, J. K., Kedl, D. M., and Shelton, J. J., "Shear Wrinkling in Isolated Spans", Proceedings of 4th International Conference on Web Handling, 1997, pp.462-479.

(3) Hashimoto, H., "Prediction Model of Paper-web Wrinkling and Some Numerical Calculation Examples with Experimental Verifications", Journal of Microsystem Technology, 13, 2007, pp.933-941. 